\title{
A UNIQUENESS THEOREM FOR ENTIRE FUNCTIONS
}

\author{
RICHARD F. DEMAR ${ }^{1}$
}

Given a sequence $\left\{\mathscr{L}_{n}\right\}$ of linear functionals defined on a linear space $C$ of functions, the uniqueness problem is to find a subspace $C_{1} \subseteq C$ such that $f$ in $C_{1}$ is uniquely determined by the sequence of numbers $\left\{\mathscr{L}_{n}(f)\right\}$; that is, $g \in C_{1}$ and $\mathscr{L}_{n}(g)=0 ; n=0,1,2, \ldots$ implies $g=0$.

We shall use the following notation. Let $K$ denote the class of all entire functions of exponential type. If $\Omega$ is a simply-connected domain in the complex plane, let $K[\Omega]$ denote the class of all $f$ in $K$ such that the Borel transform of $f$, which we shall denote by $F$, is regular on $\Omega^{\prime}$, the complement of $\Omega$. (If $f(z)=\sum a_{n} z^{n} / n$ !, then $F(\zeta)$ $=\sum a_{n} \zeta^{-n-1}$.) We shall deal with sequences $\left\{\mathscr{L}_{n}\right\}$ of linear functionals defined on a class $K[\Omega]$ by

$$
\mathcal{L}_{n}(f)=\frac{1}{2 \pi i} \int_{\Gamma}[W(\zeta)]^{n} F(\zeta) d \zeta
$$

for some function $W$ regular on $\Omega$, where $\Gamma$ is a simple contour contained in $\Omega$ and enclosing all singularities of $F$. This class of functionals has been studied by Gelfond [5], Buck [3], and the author [4]. Examples of functionals having a representation (1) are $\mathcal{L}_{n}(f)$ $=f(n), \quad \mathscr{L}_{n}(f)=\Delta^{n} f(0)$, and $\mathfrak{L}_{n}(f)=f^{(n)}(n)$ for which the functions $W(\zeta)$ are $e^{\zeta}, e^{\zeta}-1$, and $\zeta e^{\zeta}$, respectively.

Previous uniqueness theorems which apply to the class of sequences of functionals having a representation (1) have been obtained by finding a class $K[\Omega]$ of functions $f$ representable by an interpolation series having the $\mathscr{L}_{n}(f)$ as coefficients. If for all $f$ in $K[\Omega]$ this series converges to $f$ in some region or is summable by some totally regular method of summation, then $K[\Omega]$ is a uniqueness class for $\left\{\mathscr{L}_{n}\right\}$. The best such sufficient condition was obtained by Buck [3] using Mittag-Leffler summability. It states that if $\Omega$ contains the origin, $W(0)=0, W$ is univalent on $\Omega$ and maps $\Omega$ onto a set which is starshaped with respect to the origin, then $K[\Omega]$ is a uniqueness class for $\left\{\mathscr{L}_{n}\right\}$. Gelfond [5] showed that the condition that $W$ is univalent on $\Omega$ is necessary for $K[\Omega]$ to be a uniqueness class for $\left\{\mathcal{L}_{n}\right\}$. We show

Presented to the Society, January 25, 1964, under the title $A$ uniqueness theorem; received by the editors September $9,1963$.

1 This work was done during the author's tenure of a National Academy of Sciences-National Research Council Postdoctoral Resident Research Associateship at the National Bureau of Standards while on leave from Miami University. 
that this one condition is also sufficient. The proof is simple and does not use interpolation series. It is similar to a proof given by Boas and Buck [2, p. 26] of a theorem on sources of nontrivial representations of zero.

THEOREM. Let $\Omega$ be a simply-connected domain and let $W$ be regular on $\Omega$. Let $\mathfrak{L}_{n}$ be defined on $K[\Omega]$ by (1) with this $W$. Then a necessary and sufficient condition for $K[\Omega]$ to be a uniqueness class for $\left\{\mathscr{L}_{n}\right\}$ is that $W$ be univalent on $\Omega$.

Proof. We give Gelfond's proof of necessity. If $W$ is not univalent on $\Omega$, then there exist $\zeta_{1}, \zeta_{2}$ in $\Omega$ such that $\zeta_{1} \neq \zeta_{2}$, but $W\left(\zeta_{1}\right)=W\left(\zeta_{2}\right)$. Let $f(z)=e^{\zeta_{1} z}-e^{\zeta_{2} z}$. Then $\mathfrak{L}_{n}(f)=\left[W\left(\zeta_{1}\right)\right]^{n}-\left[W\left(\zeta_{2}\right)\right]^{n}=0 ; n=0,1$, $2, \cdots$, but $f \neq 0$.

To prove sufficiency, let $W$ be univalent on $\Omega$ and let $f$ in $K[\Omega]$ satisfy $\mathfrak{L}_{n}(f)=0 ; n=0,1,2, \cdots$, i.e.,

$$
\int_{\Gamma}[W(\zeta)]^{n} F(\zeta) d \zeta=0, \quad n=0,1,2, \cdots
$$

Let $\Omega_{w}$ be the image of $\Omega$ and $\Gamma_{w}$ the image of $\Gamma$ under $w=W(\zeta)$. Since $W$ is regular and univalent on $\Omega, \Gamma_{w}$ is a simple contour enclosing the image of the region enclosed by $\Gamma$. Let $\zeta=Z(w)$ be the inverse of $W$ which maps $\Omega_{w}$ onto $\Omega$. Then $Z$ is regular and univalent on $\Omega_{w}$; so $Z^{\prime}(w) \neq 0$ on $\Omega_{w}$. Since $\Gamma$ encloses all singularities of $F(\zeta), \Gamma_{w}$ encloses all singularities of $F(Z(w))$; so, $F(Z(w))$ is analytic on $\Gamma_{w}$. Substituting $\zeta=Z(w)$ in the integrals, we obtain

$$
\int_{\Gamma_{w}} w^{n} F(Z(w)) Z^{\prime}(w) d w=0, \quad n=0,1,2, \cdots
$$

Multiplying the $n$th integral by $z^{n} / n$ ! and summing, we obtain

$$
\int_{\Gamma_{w}} e^{z w} F(Z(w)) Z^{\prime}(w) d w=0
$$

for all $z$. Since $F(Z(w)) Z^{\prime}(w)=G(w)$ is analytic on $\Gamma_{w}$, this implies, by a lemma of Pólya $[1$, p. 110$]$, that $G$ is analytic inside $\Gamma_{w}$. Then, since $Z^{\prime}(w)$ is analytic and nonzero inside $\Gamma_{w}, F(Z(w))=G(w) / Z^{\prime}(w)$ is analytic inside $\Gamma_{w}$. Since $Z$ is analytic inside $\Gamma_{w}$, this implies $F$ is analytic inside $\Gamma$. But $F$ is also analytic outside and on $\Gamma$ and at $\infty$; so $F$ is a constant. Since $F(\infty)=0, F=0$, and this implies $f=0$, which completes the proof.

A simple example of a specialization of the theorem is that if $\Omega$ is a simply-connected domain, then $K[\Omega]$ is a uniqueness class for 
either $\{f(n)\}$ or $\left\{\Delta^{n} f(0)\right\}$ if and only if $\zeta \in \Omega$ implies $(\zeta+2 n \pi i) \notin \Omega$, $n=1,2, \cdots$. From this, dropping the requirement that $G$ must be open in the notation $K[G]$, the class $K\left[\Omega_{1}\right]$ is a uniqueness class for either of these sequences of functionals if $\Omega_{1}$ is the strip $\{x+i y \mid$ $-\pi<y \leqq \pi\}$. The class $K\left[\Omega_{1}\right]$ is maximal in the sense that if $\Omega_{2}$ is any set which properly contains $\Omega_{1}$, then $K\left[\Omega_{2}\right]$ is not a uniqueness class. That $K\left[\Omega_{1}\right]$ is a uniqueness class follows from the fact that if the Borel transform of a function $f$ is analytic on the complement of $\Omega_{1}$, then it is analytic on the complement of some open horizontal strip of width $2 \pi$.

\section{BibLIOGRAPHy}

1. R. P. Boas, Entire functions, Academic Press, New York, 1954.

2. R. P. Boas and R. C. Buck, Polynomial expansions of analytic functions, Springer, Berlin, 1958.

3. R. C. Buck, Interpolation series, Trans. Amer. Math. Soc. 64 (1948), 283-298.

4. R. F. DeMar, Existence of interpolating functions of exponential type, Trans. Amer. Math. Soc. 105 (1962), 359-371.

5. A. O. Gelfond, Interpolation et unicite des fonctions entières, Rec. Math. Moscow (N.S.) 4 (1938), 115-147.

\section{Miami University and}

National Bureau of Standards 